\title{
Evaluación de la diversidad gama de corales arrecifales (Scleractinia) en el Pacífico de México
}

\section{Evaluation of gamma diversity of reef corals (Scleractinia) in the Mexican Pacific}

\author{
Héctor Reyes-Bonilla ${ }^{1}$, Luis E. Calderón-Aguilera2 ${ }^{2 *}$, Gabriela Cruz-Piñón ${ }^{2,5}$, Ramón Andrés López-Pérez ${ }^{3}$ y \\ Pedro Medina-Rosas ${ }^{4}$ \\ ${ }^{1}$ Departamento de Biología Marina, Universidad Autónoma de Baja California Sur. Apartado postal 19-B, La Paz 23080, Baja California Sur, \\ México. \\ ${ }^{2}$ Centro de Investigación Cientifica y de Educación Superior de Ensenada. Km. 107 Carretera Tijuana-Ensenada, Ensenada 22860, Baja California, \\ México. \\ ${ }^{3}$ Universidad del Mar, Instituto de Recursos. Apartado postal 47, Puerto Ángel 70902, Oaxaca, México. \\ ${ }^{4}$ Universidad de Guadalajara, Centro Universitario de la Costa. Av. UdeG 203, Delegación Ixtapa, Puerto Vallarta 48280, Jalisco, México. \\ ${ }^{5}$ University of Sheffield, Department of Animal and Plant Sciences, Alfred Denny Building. Western Bank, Sheffield S10 2TN, United Kingdom. \\ *Correspondencia: leca@cicese.mx
}

\begin{abstract}
Resumen. Con el fin de evaluar el conocimiento de la diversidad regional de corales zooxantelados, se aplicaron métodos de máxima verosimilitud (chao, jacknife) y remuestreo (bootstrap) sobre una base de datos de 3423 registros, ordenada en 15 áreas geográficas del Pacífico mexicano. Los análisis se hicieron para la riqueza específica total (34 especies) y las 5 familias de corales conocidas y se contrastaron con los valores esperados, considerando todas las especies reportadas para el Pacífico oriental. La riqueza conocida de especies de corales zooxantelados posiblemente esté subestimada hasta en un 25\%, al igual que en el caso de las familias Pocilloporidae, Poritidae y Agariciidae, mientras que para Siderastreidae y Fungiidae no hay diferencias entre los valores esperados y los observados. Considerando el área de distribución de las especies raras en el litoral oeste de México y el historial de registros, se sugiere que las próximas especies a encontrarse posiblemente aparezcan en la zona de las islas Revillagigedo, en la parte sur de la península de Baja California o la zona continental adyacente (Sonora) y en las costas de Oaxaca. Este tipo de análisis son fundamentales para la optimización del esfuerzo de muestreo y para el establecimiento de áreas naturales protegidas que salvaguarden la biodiversidad conocida y potencial.
\end{abstract}

Palabras clave: biodiversidad, métodos no paramétricos, máxima verosimilitud, bootstrap.

\begin{abstract}
In order to assess gamma diversity of hermatypic corals, maximum likelihood methods (Chao, Jacknife) and resampling (bootstrap) were used over a database with 3423 records of corals from 15 regions of the Mexican Pacific. Analysis were conducted for the species richness ( 34 species) and for the 5 families reported. Results suggest that species richness might be underestimated up to $25 \%$ as well as for the families Pocilloporidae, Poritidae, and Agariciidae. On the other hand, richness observed for Siderastreidae and Fungiidae is not significantly different from expected. According to rare species distribution and historical records in the Mexican Pacific, it is expected that new records will come either from the Revillagigedo, southern region of the Baja California Peninsula or adjacent mainland (Sonora) and Oaxaca coast. This kind of analysis is fundamental in order to optimize sampling efforts and to establish marine protected areas that protect known and potential species richness.
\end{abstract}

Key words: biodiversity, non-parametric methods, maximum likelihood, bootstrap.

\section{Introducción}

La biodiversidad representa la variedad de la vida en todos los niveles jerárquicos, desde el gen hasta el ecosistema (Wilson, 1988). Es éste un concepto atractivo e incluyente; sin embargo, la complejidad de los ecosistemas hace que el cálculo real de la biodiversidad sea impracticable, y que

Recibido: 09 diciembre 2008; aceptado: 09 octubre 2009 se busquen medidas paralelas que ayuden a comprender la estructura y funcionamiento de las comunidades o ensamblajes (Gaston y Spicer, 2004). En este sentido, la riqueza de especies ha sido considerada como el mejor indicador proximal de la diversidad biológica dado que sus valores son intuitivamente comprensibles y fácilmente apreciables por el público y los tomadores de decisión (haciéndola valiosa para fines de conservación y manejo; Gaston, 2000), y además es la base para el conocimiento 
de las tendencias macroevolutivas e historial de los taxa superiores (Halffter y Moreno, 2005). La evaluación adecuada del número de especies también es relevante para los escenarios de cambio global, donde la invasión, extinción y cambios en la distribución de especies serán fenómenos frecuentes (Renema et al., 2008).

La generación de inventarios completos de taxa individuales presentes en una zona dada no es sencilla debido a la enorme inversión que se requiere en forma de tiempo y recursos (materiales, humanos, económicos); este tipo de problemas se agrava cuando se analizan especies marinas, ya que los costos se elevan sensiblemente (Gray, 2001). Para solventar el problema se han adoptado estrategias como la generación de curvas de acumulación de especies, donde se ajusta la tendencia de la riqueza a modelos no lineales que presentan asíntotas y a partir de unidades de esfuerzo definidas (años de trabajo, número de publicaciones, etc.; Colwell y Coddington, 1994; Soberón et al., 2007). Dado que en muchas ocasiones los datos acumulados de riqueza no pueden ser ajustados a las curvas, la alternativa principal a dicho procedimiento la ofrecen los métodos no paramétricos de estimación, también llamados "no asintóticos" (Moreno, 2001; Gotelli y Colwell, 2001), lo que implica que la cifra observada de número de especies presentes es representativa y sirve de marco de referencia para estimar el total de taxa en la zona de estudio; dicho valor se obtiene sumando a la riqueza observada un factor de corrección que representa el "número de especies faltantes" y que se calcula con base en las características de la frecuencia con que ocurren las especies, o mediante técnicas de remuestreo de los datos (Willott, 2001). El primer grupo de técnicas para saber la diferencia entre la riqueza observada y la potencialmente presente se basa conceptualmente en los análisis de marcado-recaptura (Chao et al., 2007), y genera sus predicciones con base en el número de especies raras en el inventario (aquellas que sólo aparecen 1 o 2 veces en las unidades muestrales), y empleando algoritmos de máxima verosimilitud. En resumen, se efectúan miles de muestreos aleatorios de subgrupos de información (normalmente de los censos que fundamentan el estudio) a partir de donde se evalúa el número esperado de especies faltantes, y de ahí se toma aquel con mayor probabilidad de que ocurra (Johnson y Omlad, 2004). En contraste, los procedimientos de remuestreo (bootstrap) sirven para calcular el número total de especies por medio de la reconstrucción de la distribución muestral del estimador; en este caso, el número de taxa no encontrados en los censos (Magurran, 2004). Para ello, de la muestra original se toman elementos de manera individual y con reemplazamiento, y con ellos evalúan la riqueza total con base en los valores promedio de dicha distribución (Rodgers, 1999).
Una de las aplicaciones de los métodos no paramétricos es la evaluación de la riqueza en regiones biogeográficas, ecorregiones o países, condición que por su gran escala espacial implica el cálculo de la diversidad gama(Whittaker, 1972). El conocimiento de la diversidad regional permite entender aspectos de la historia biogeográfica y de los procesos ecológicos ligados principalmente a los hábitats y factores ambientales (Arellano y Halffter, 2003), y es clave para la planeación de estrategias de conservación del tipo de las redes de áreas protegidas (Roberts et al., 2002). En la literatura sobre especies marinas pueden hallarse evaluaciones del total de la riqueza mundial o de algunos océanos para peces o gasterópodos (Vermeij, 1999; Zapata y Robertson, 2005), pero el tema aún no ha sido explorado con suficiente profundidad ya que hay limitada disponibilidad de información sobre muchos taxa (Cairns, 2007).

El Pacífico oriental tropical es una de las partes del planeta donde existen condiciones aceptables para el estudio de la diversidad gama de muchos grupos, dados los intensos esfuerzos que han realizado expediciones extranjeras e instituciones nacionales desde hace más de un siglo (Hendrickx et al., 2005) y, de esa región, uno de los taxa mejor caracterizados es el de los corales zooxantelados, también llamados arrecifales o hematípicos (Reyes Bonilla y López Pérez, 1998; Reyes Bonilla, 2002), los cuales han llamado la atención porque forman arrecifes que concentran un gran número de especies endémicas y colonizadoras del Indo Pacífico (Glynn y Ault, 2000), y están expuestos a altos niveles naturales de baja alcalinidad como resultado de las intensas surgencias que se presentan en toda la región (Cortés, 1997). Esta situación los hace modelos idóneos para evaluar los cambios potenciales en la función de los ecosistemas coralinos en el futuro (Hoegh-Guldberg et al., 2007). Finalmente, la fauna de corales zooxantelados tiene una historia evolutiva común (López Pérez, 2005) y en el oeste de México se distribuye exclusivamente dentro de 2 regiones biogeográficas (Corteziana y Mexicana; Glynn y Ault, 2000), y 4 ecoregiones bien definidas (Corteziana, Transición Magdalena, Pacífico tropical mexicano e islas Revillagigedo; Spalding et al., 2007), por lo que el país representa una unidad válida para el análisis de la diversidad gama.

El objetivo del presente estudio fue evaluar qué tan completo está el elenco de especies de corales zooxantelados (es decir, el valor de diversidad gama), mediante el uso de métodos no paramétricos de estimación. Los resultados indican que el elenco de especies conocido aún está incompleto, aunque es probable que al menos para un par de familias, en efecto, ya conozcamos la riqueza total. 


\section{Materiales y métodos}

En este trabajo partimos de la información disponible en la publicación de Reyes Bonilla et al. (2005), donde se presentan más de 3000 registros individuales y georeferenciados de todas las especies de corales escleractinios zooxantelados y azooxantelados del occidente de México, con precisión de grados, minutos y segundos. La parte de los corales arrecifales de dicha base de datos se ensambló a partir de extenso trabajo de campo entre 1989 y 2004, datos de ejemplares revisados en más de una docena de colecciones de museos en México y en el extranjero, consultas con especialistas, y revisión de toda la literatura disponible sobre el tema entre 1848 y 2004 (casi 100 trabajos). Para fines de este trabajo la información se ordenó de acuerdo con las 15 áreas geográficas sugeridas por Reyes Bonilla y López Pérez (1998; Fig. 1), que fueron definidas con base en su similitud en composición de especies coralinas.

A partir de la matriz de datos (15 localidades $x$ 34 especies) se estimó la diversidad gama de taxa zooxantelados en el Pacífico mexicano, en total y para cada familia, por suma directa de las especies registradas. Posteriormente, para saber si dichas cifras coincidían o no con los valores esperados de riqueza total en la región, se aplicaron los modelos de Chao, Chao 2, jacknife, jacknife 2 (máxima verosimilitud) y bootstrap (remuestreo, utilizando 1000 iteraciones), mediante el programa PRIMER, ver. 6.0 (véase ecuaciones en Colwell y Coddington, 1994; Moreno, 2001; Magurran, 2004, entre otras publicaciones). Esas técnicas fueron elegidas debido a que funcionan con información sobre presencia y ausencia de especies, y porque han demostrado ser estimadores precisos de la riqueza (Willott, 2001). Una vez que se obtuvieron los estimadores de la riqueza total y por familia, se analizó estadísticamente la diferencia entre la riqueza observada y la propuesta por cada modelo. Para la comparación se aplicó primero el método de Sokal y Rohlf (1995), el cual sirve para comparar valores individuales de parámetros estimados, contra una serie de datos esperados que se supone siguen un comportamiento normal. El procedimiento emplea una prueba $t$ de Student modificada, que en este caso tomó en cuenta la riqueza total cuantificada de la base de datos, y los valores de la media y la desviación típica de los 5 estimadores de riqueza. Este tipo de técnicas, donde el valor esperado de un parámetro ecológico es comparado con el del promedio de una serie de estimadores, han sido recomendadas cuando ninguno de los procedimientos es cualitativamente superior a otro y por ello se considera que el promedio de esa serie de valores es un estimador potencialmente más robusto que cualquiera de modo individual (Johnson y Omland, 2004). Para realizar una segunda comparación entre la riqueza observada y la esperada, pero suponiendo una distribución libre, se utilizó un método de remuestreo donde se generaron 1000 valores esperados de la diversidad gama total y por familia, a partir de los valores esperados de riqueza arrojados por los 5 métodos empleados. Luego se evaluó de manera directa la probabilidad de que ocurra el valor real registrado.

\section{Resultados}

Los análisis indicaron que las 34 especies que componen la riqueza de corales zooxantelados registrada en la actualidad en el Pacífico mexicano es probablemente una subestimación del valor real. Las estimaciones arrojadas por los métodos no paramétricos indican que el valor de los estimadores siempre es superior al valor observado, variando

Figura 1. Zonas de estudio en el Pacífico mexicano. 1, bahía Magdalena; 2, Baja California; 3, Sonora; 4, Baja California Sur zona Norte; 5, Baja California Sur zona Centro; 6, Baja California Sur zona Sur; 7, Sinaloa; 8 , islas Marías; 9, Nayarit; 10, Jalisco; 11, islas Revillagigedo; 12, Colima; 13. Michoacán; 14, Guerrero; 15, Oaxaca. 
entre 37.8 (bootstrap) hasta 46.9 especies (jacknife 2), con un promedio de $43.09 \pm 1.51$ (parámetro \pm error típico; Cuadro 1), lo que sugiere que en la región de estudio faltan por encontrarse unas 9 especies de este grupo.

De manera semejante, los valores de riqueza total estimada por familia generalmente fueron inferiores a los propuestos por los modelos (Cuadro 1), aunque para la familia Fungiidae la cifra conocida en la actualidad a partir de datos de campo y literatura prácticamente coincide con la que sugieren los modelos no paramétricos. De manera semejante, para la familia Pocilloporidae y Siderastreidae tampoco hubo grandes diferencias.

El último análisis consistió en una comparación de los valores observados y esperados mediante procedimientos paramétricos (prueba $t$ de Student de comparación de observaciones individuales y datos de muestreo) y no paramétricos (bootstrap con 1000 repeticiones; Cuadro 2, Fig. 2). Ambas pruebas señalan que el número total de especies reconocidas en el Pacífico mexicano es significativamente menor al calculado por los 5 modelos, y de igual manera se encontró que para las familias Pocilloporidae, Poritidae y Agariciidae, la riqueza registrada actualmente es significativamente menor a la esperada. Sin embargo, para Siderastreidae y Fungiidae no hay diferencias entre los valores reales y los teóricos. Estos resultados sugieren que el número de corales zooxantelados que han sido identificados como residentes del Pacífico mexicano es inferior al real, es decir, existen especies que aún no han sido observadas por los investigadores o anotadas en la literatura profesional; la consecuencia del caso es que actualmente debemos tener sólo un inventario parcial de los taxa que habitan la zona. En el mismo sentido, el elenco sistemático de 3 familias residentes de la costa oeste de México (Pocilloporidae, Poritidae, Agariciidae) parece estar aún incompleto, mientras que la cifra podría ser definitiva para las 2 restantes (Siderastreidae, Fungiidae).

\section{Discusión}

El estudio de la diversidad gama de corales zooxantelados del Pacífico mexicano sugiere que el inventario actual de especies no está completo; de hecho, los modelos no paramétricos indican que la cifra puede ser hasta un $25 \%$ superior a la actual. Esta diferencia puede analizarse por 2 líneas: a) la forma en que operan los métodos estadísticos, y b) la riqueza actual del Pacífico mexicano en comparación con la riqueza de especies de corales en el Pacífico oriental, misma que puede tomarse como un valor máximo contra el cual comparar los resultados de las estimaciones de los modelos aplicados.

Los modelos no paramétricos de riqueza de especies responden a la frecuencia con la que las especies raras aparecen en la matriz de datos, es decir, aquellas que están presentes en 1 o 2 localidades (Colwell y Coddington, 1994). Lo anterior resulta debido a que el algoritmo parte del supuesto que la frecuencia de especies raras disminuirá mientras el muestreo sea más eficiente, aunque en teoría nunca llegará a cero; así, mientras menor sea el número de especies raras en la matriz de datos, más cerca se estará de la estimación correcta de la riqueza (Magurran, 2004). En el caso de los corales del Pacífico mexicano, el Apéndice 1 muestra que $23 \%$ de las especies pueden considerarse raras pues habitan una sola región en el país, lo que dio lugar a que los estimadores de riqueza empleados consideren que la fauna actual representa, en el peor de los escenarios, sólo el $75 \%$ de la riqueza potencial del área (Cuadro 1; Fig, 2). Resulta relevante que las especies consideradas raras en el Pacífico mexicano, comúnmente se encuentran restringidas a las islas Revillagigedo. Este archipiélago cuenta con una gran riqueza específica y un alto nivel de endemismo en sus faunas marinas (Bautista Romero et al., 1994), pero además ha llamado la atención de los zoogeógrafos por ser uno de los puntos más importantes de colonización de especies desde el Pacífico central (Cortés, 1997). La zona recibe la influencia anual de una lengua de agua proveniente de la contracorriente ecuatorial que viene del sur y que es particularmente fuerte en años cuando ocurre la oscilación sureña de El Niño; por esa razón, se ha sugerido que es durante estos eventos cuando se han llevado a cabo la mayor parte de los procesos de colonización (Ketchum y Reyes Bonilla, 1997; Glynn y Ault, 2000). El hecho de que varios corales poco comunes

Cuadro 1. Riqueza observada de corales zooxantelados en el Pacífico mexicano (número de especies total y por familia; equivalente a la diversidad gama), y riqueza esperada según cada uno de los modelos de estimación no paramétricos

\begin{tabular}{lcccccc}
\hline Modelo & Total & Pocilloporidae & Poritidae & Agariciidae & Siderastreidae & Fungiidae \\
\hline Observado & 34 & 9 & 8 & 8 & 6 & 3 \\
Chao 1 & 44.13 & 9.5 & 12.5 & 12.5 & 6 & 3 \\
Chao 2 & 44.13 & 9.5 & 12.5 & 12.5 & 6 & 3 \\
Jacknife 1 & 42.4 & 9.93 & 10.8 & 10.8 & 7.87 & 3 \\
Jacknife 2 & 46.98 & 9.99 & 12.59 & 12.59 & 9.6 & 2.2 \\
Bootstrap & 37.83 & 9.51 & 9.19 & 9.23 & 6.78 & 3.12 \\
\hline
\end{tabular}


Cuadro 2. Riqueza de especies de coral, total y por familia, registradas en el Pacífico mexicano. Las cifras arrojadas por los cinco modelos de estimación de diversidad gama se confrontó con los valores registrados mediante una prueba de $t$ de Student (paramétrica) y una de remuestreo (no paramétrica). Los valores en negritas indican significancia estadística ( $>0.05$ )

\begin{tabular}{lcccc}
\hline Taxon & $N$ & $\begin{array}{c}\text { Promedio (error típico) } \\
\text { de los valores esperados }\end{array}$ & Prueba de & $\begin{array}{c}\text { Bootstrap P }(1,000 \\
\text { simulaciones) }\end{array}$ \\
\hline Riqueza total & 34 & $43.092(1.507)$ & $\mathbf{0 . 0 0 3}$ & $\mathbf{0 . 0 0 4}$ \\
Pocilloporidae & 9 & $9.687(0.114)$ & $\mathbf{0 . 0 0 3}$ & $\mathbf{0 . 0 2 4}$ \\
Poritidae & 8 & $11.525(0.665)$ & $\mathbf{0 . 0 0 4}$ & $\mathbf{0 . 0 1 5}$ \\
Agariciidae & 8 & $11.518(0.671)$ & 0.004 & 0.240 \\
Siderastreidae & 6 & $7.249(0.679)$ & 0.981 & 0.792 \\
Fungiidae & 3 & $2.862(0.168)$ & \\
\hline
\end{tabular}

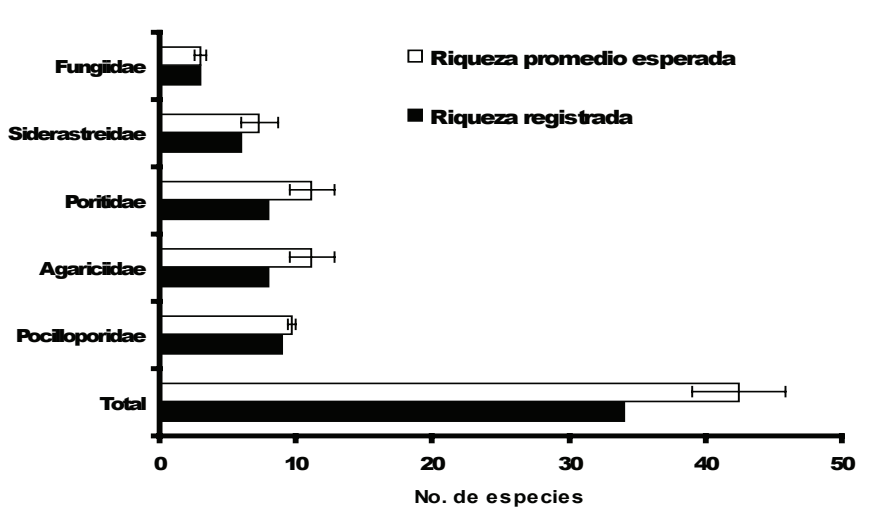

Figura 2. Comparación del valor de riqueza de especies observado y el promedio de los valores esperados $( \pm$ desviación típica), según los 5 modelos.

que habitan las Revillagigedo no estén en el macizo continental está claramente documentado (Reyes Bonilla y López Pérez, 1998; Ketchum y Reyes Bonilla, 2001) y es importante señalar que el fenómeno de la colonización a larga distancia desde el Pacífico central ha aumentado notablemente el número de especies registradas en el occidente de México (Reyes Bonilla et al., 2005).

Para conocer qué tan precisas son las estimaciones aquí presentadas de la diversidad gama de los corales, es ilustrativo contrastar los valores de riqueza arrojados por los modelos contra un límite máximo conocido. En este caso, dicho indicador proximal estuvo representado por el número de especies presentes en la costa occidental del continente americano, pero que no se encuentran al norte de los $11^{\circ}$ de latitud norte (costa de El Salvador). Esta suposición es válida por la cercanía espacial entre las regiones y porque existen muchos taxa en común, lo que garantiza un cierto nivel de intercambio faunístico (Glynn y Ault, 2000), aunque en realidad debe tomarse como una leve subestimación al considerar que el Pacífico central también es una fuente potencial de entrada de especies de corales zooxantelados a México, vía el atolón Clipperton y las islas Revillagigedo (Ketchum y Reyes Bonilla, 2001). Como se ve en el Cuadro 3, hay 11 especies arrecifales en Centro y Sudamérica que no han sido registradas en México, y la mayoría de ellas reside en Costa Rica, otra de las zonas principales que recibe inmigración desde el oeste (Cortés, 1997). Las discrepancias interregionales se deben fundamentalmente a la presencia de múltiples especies de los géneros Leptoseris y Pavona al sur del continente, ambos pertenecientes a la familia Agariciidae.

Por otra parte, el número total de especies zooxanteladas en el Pacífico oriental tropical es de 45 (Reyes Bonilla, 2002); esta marca puede considerarse el extremo superior de riqueza que es posible encontrar en México, y es la base para el siguiente análisis.

Según los datos de la riqueza total esperada de corales en el oeste del país (Cuadro 1), sólo un modelo (jacknife 2) propone una cifra superior a la citada (46.9 especies); los demás fluctúan entre 37.8 (bootstrap) y 44.1 (chao 1). En este sentido, puede sugerirse que los valores calculados de diversidad gama coralina en el Pacífico mexicano son robustos, ya que los modelos no sobrestimaron el valor máximo de riqueza que potencialmente puede ocurrir en el área (45 especies). La última observación dentro de este tópico es que el valor promedio de 43 especies predichas por los 5 métodos indicaría que la riqueza de corales en México prácticamente equivaldría a la conocida para la región del Pacífico oriental tropical. La evidencia empírica indica que, en efecto, es muy difícil que en México existan poblaciones de todos los taxa, ya que al menos hay 3 especies cuya probabilidad de encontrarse es muy baja: Acropora valida y Porites rus fueron vistas sólo en una ocasión en Costa Rica y Colombia (luego del ENSO [El Niño Southern Oscillation] de 1987), y sus poblaciones aparentemente se extinguieron en la región para finales de la década de 1990 (Cortés, 1997). La tercera especie, Siderastrea glynni, es una endémica de Panamá que se 
Cuadro 3. Especies de corales zooxantelados no presentes en México, pero que residen en alguna otra región del Pacífico oriental tropical*

\begin{tabular}{lll}
\hline Familia & Especie** & Otros países donde se ha observado en la costa americana \\
\hline Pocilloporidae & Pocillopora danae & Chile (isla de Pascua) \\
Acroporidae & Acropora valida & Colombia \\
Poritidae & Porites rus & Costa Rica \\
Agariciidae & Pavona frondifera & Costa Rica y Panamá \\
& Pavona xarifae & Endémico de Costa Rica \\
& Pavona chiriquiensis & Costa Rica, Panamá, Colombia y Ecuador (continente e islas Galápagos) \\
& Leptoseris foliosa & Ecuador (islas Galápagos) \\
& Leptoseris hawaiiensis & Costa Rica \\
& Leptoseris scabra & Costa Rica, Ecuador y el atolón Clipperton \\
& Leptoseris solida & Chile (isla de Pascua) \\
Siderastreidae & Siderastrea glynni & Endémico de Panamá \\
\hline
\end{tabular}

*Datos de Reyes Bonilla (2002), Reyes Bonilla et al. (2005) y Carpenter et al. (2008).

**Especies inmigrantes del Pacífico central y occidental, excepto cuando se especifica.

encuentra en situación crítica (menos de 50 colonias en total; Carpenter et al., 2008), por lo que difícilmente será capaz de colonizar hacia el norte en un futuro próximo, o tener poblaciones actuales.

Siguiendo esta línea de análisis, pero en el nivel de familia, es interesante anotar que para Fungiidae el elenco sistemático encontrado en México es idéntico al del Pacífico oriental en su totalidad (3 especies; Reyes Bonilla, 2002), mientras que para Siderastreidae sólo falta una (la citada Siderastrea glynni), y al mismo tiempo ningún modelo de distribución libre generó estimadores muy distintos a los valores reales (cifras máximas de 3.11 especies en Fungiidae según bootstrap, y 9.61 para Siderastreidae según jacknife 2). De esta forma, puede decirse que el análisis numérico confirma los estudios de campo. En claro contraste, para Agariciidae y Poritidae las cifras esperadas son mucho mayores que las observadas (Cuadro 1; Fig. 2), y prácticamente en todos los casos los estimadores sugieren una riqueza total superior a las 10 especies (un $20 \%$ mayor al actual de 8). Como se indicó en el caso de la estimación del total de las especies, la explicación de las diferencias entre lo observado y lo esperado está en la presencia de especies raras en ambas familias, en su mayoría presentes en las Revillagigedo y Oaxaca (Apéndice 1); además, para Agariciidae, dichas discrepancias pueden ser reflejo de que esta familia tiene un alto número de representantes que no se distribuyen en México (Cuadro 3), y que quizá eventualmente lleguen a colonizar la región. Esta sugerencia se hace al considerar que de hecho 6 de las 8 especies reconocidas en la actualidad en el Pacífico mexicano no habían sido registradas a mediados de los años noventa (Reyes Bonilla y López Pérez, 1998; Leyte Morales et al., 2001; Reyes Bonilla et al., 2005). Tomando en conjunto la evidencia numérica y la observacional, es posible que en realidad para esta familia aún haya varias especies por ser descubiertas en México.

La conclusión principal de este trabajo es que la riqueza de especies de corales zooxantelados conocida en el Pacífico mexicano posiblemente sea una subestimación del valor real. Lo incompleto del elenco sistemático de este taxon tiene consecuencias interesantes desde varias perspectivas. Inicialmente, implica que el trabajo de ciencia básica en el litoral oeste de México aún está lejos de concluirse y que se requieren expediciones a localidades poco conocidas y caracterizadas como la costa entre Colima y Michoacán (Reyes Bonilla et al., 2005). Sin embargo, es esencial revisar en detalle las islas Revillagigedo, el sitio por excelencia para la entrada de taxa inmigrantes del oeste (Bautista Romero et al., 1994). Considerando las opiniones vertidas en otros trabajos similares (Zapata y Robertson, 2005), las especies de corales aún no detectadas deben tener un ámbito restringido de distribución y quizá ocupen hábitats particulares. Un ejemplo de ello está en Leptoseris papyracea, un agaricido que en México reside sobre fondos arenosos o en mantos de rodolitos, y a profundidades superiores a los $-10 \mathrm{~m}$ (Leyte Morales et al., 2001). Finalmente, y desde la óptica de la conservación, podría decirse que la determinación de sitios prioritarios se ve afectada por las condiciones del relativo desconocimiento de cuáles son las especies más raras, y dónde están las zonas en las que se encuentran. Este punto es relevante si se considera la necesidad de establecer áreas protegidas en los litorales de Colima, Michoacán y Guerrero, donde no se tiene ninguna. 


\section{Agradecimientos}

A los colegas, amigos y estudiantes que han participado con nosotros en más de una década de visitas de campo a todos los estados de la costa oeste de México. Publicación derivada de los proyectos CONACyT 37528-B SEMARNAT-CONACyT 0189 y CONABIO AS07. Los comentarios de 2 revisores contribuyeron a mejorar sensiblemente la calidad de la versión final del manuscrito.

\section{Literatura citada}

Arellano, L. y G. Halffter. 2003. Gamma diversity derived from and a determinant of alpha diversity and beta diversity. An analysis of three tropical landscapes. Acta Zoológica Mexicana (n. s.) 090:27-76.

Bautista Romero, J., H. Reyes Bonilla, D. Lluch Cota y S. Lluch Cota. 1994. Aspectos generales de la fauna marina. In La isla Socorro, Reserva de la Biosfera Archipiélago de Revillagigedo, A. Ortega Rubio y A. Castellanos Vera (eds.), Centro de Investigaciones Biológicas del Noroeste (CIBNOR), La Paz, Baja California Sur. p. 243-275.

Cairns, S. D. 200. Deep-water corals: an overview with special reference to diversity and distribution of deep water scleractinian corals. Bulletin of Marine Science 81:311-322.

Carpenter K. E, M. Abrar, G. Aeby, R. B. Aronson, S. Banks, A. Bruckner, A. Chiriboga, J. Cortés, J. C. Delbeek, L. De Vantier, G. J. Edgar, A. J. Edwards, D. Fenner, H. M. Guzmán, B. W. Hoeksema, G. Hodgson, O. Johan, W. Y. Licuanan, S. R. Livingstone, E. R. Lovell, J. A. Moore, D. O. Obura, D. Ochavillo, B. A. Polidoro, W. F. Precht, M. C. Quibilan, C. Reboton, Z. T. Richards, A. D. Rogers, J. Sanciangco, A. Sheppard, C. Sheppard, J. Smith, S. Stuart, E. Turak, J. E. N. Veron, C. Wallace, E. Weil y E. Wood. 2008. One-third of reef-building corals face elevated extinction risk from climate change and local impacts. Science 321:560-563.

Chao, A., R. K. Colwell, C. W. Lin y N. J. Gotelli. 2009. Sufficient sampling for asymptotic minimum species richness estimations. Ecology 40:1125-1133.

Colwell, R. K. y J. A. Coddington. 1994. Estimating terrestrial biodiversity through extrapolation. Philosophical Transactions of the Royal Society 345:101-118.

Cortés, J. 1997. Biology and geology of eastern Pacific coral reefs. Coral Reefs 16, suppl. S39-S46.

Gaston, K. J. 2000. Global patterns in biodiversity. Nature 405:220-227.

Gaston, K. J. y J. I. Spicer. 2004. Biodiversity: an introduction, 2nd edition, Blackwell, Oxford.

Glynn P. W. y J. S. Ault, 2000. A biogeographic analysis and review of the far eastern Pacific coral reef region. Coral Reefs 19:1-23.

Gray, J. S. 2001. Marine diversity: the paradigms in patterns of species richness examined. Scientia Marina 65, suppl. 2:4156.

Halffter, G. y C. E. Moreno. 2005. Significado biológico de las diversidades alfa, beta y gama. In Sobre la diversidad biológica: el significado de las diversidades alfa, beta y gama, G. Halffter, J. Soberón, P. Koleff y A. Meliá (eds.). M3M: Vol. 4, SEA, Zaragoza. p. 5-18.

Hendrickx, M. E., R. C. Brusca y L. T. Findley (eds.) 2005. Listado y distribución de la macrofauna del golfo de California, México, Parte I. Invertebrados. Arizona-Sonora Desert Museum/Conservation International, Tucson. 429 p.

Hoegh-Guldberg, O., P. J. Mumby, A. J. Hooten, R. S. Steneck, P. Greenfield, E. Gomez, C. D. Harvell, P. F. Sale, A. J. Edwards, K. Caldeira, N. Knowlton, C. M. Eakin, R. Iglesias-Prieto, N. Muthiga, R. H. Bradbury, A. Dubi, M. E. Hatziolos. 2007. Coral reefs under rapid climate change and ocean acidification. Science 318:1737-1742.

Johnson, J.B. y K. S. Omland. 2004. Model selection in ecology and evolution. Trends in ecology and evolution 19:101-108.

Ketchum, J. T. y H. Reyes Bonilla. 1997. Biogeography of hermatypic corals from the Revillagigedo Archipelago, Mexico. Proceedings of the $8^{\text {th }}$ International Coral Reef Symposium, Panama 1:471-476.

Leyte Morales, G. E., H. Reyes Bonilla, C. E. Cintra Buenrostro y P. W. Glynn. 2001. Range extension of Leptoseris papyracea (Scleractinia) to the west coast of México. Bulletin of Marine Science 69:1233-1237.

López Pérez, R. A. 2005. The Cenozoic hermatypic corals in the eastern Pacific: history of research. Earth Science Reviews 72:67-87

Moreno, C. E. 2001. Métodos para medir la biodiversidad, vol.1. M\&T-Manuales y Tesis SEA, Zaragoza. $84 \mathrm{p}$.

Moreno, C. E. y G. Halffter. 2000. Assessing the completeness of bat biodiversity inventories using species accumulation curves. Journal of Applied Ecology 37:149-158.

Renema, W., D. R. Bellwood, J. C. Braga, K. Bromfield, R. Hall, K. G. Johnson, P. Lunt, C. P. Meyer, L. B. McMonagle, R. J. Morley, A. O’Dea, J. A. Todd, F. P. Wesselingh, M. E. J. Wilson, J. M. Pandolfi. 2008. Hopping hotspots: global shifts in marine biodiversity. Science 321:654-657.

Reyes Bonilla, H. 2002. Checklist of valid names and synonyms of stony corals (Anthozoa: Scleractinia) from the eastern Pacific. Journal of Natural History 36:1-13.

Reyes Bonilla, H., L. E. Calderón Aguilera, G. Cruz Piñón, P. Medina Rosas, A. López Pérez, D. Herrero, G. Leyte Morales, A. Cupul y J. Carriquiry. 2005. Atlas de los corales pétreos del Pacífico mexicano. CICESE/CONABIO/CONACYT/ UABCS/UdG/UMAR, México.124 p.

Reyes Bonilla, H. y A. López Pérez. 1998. Biogeografía de los corales pétreos (Anthozoa: Scleractinia) del Pacífico de México. Ciencias Marinas 24:211-224.

Roberts, C. M., C. J. McClean, J. E. N. Veron, J. P. Hawkins, G. R. Allen, D. E. McAllister, C. G. Mittermeier, F. W. Schueler, M. Spalding, F. Wells, C. Vynne, T. B. Werner. 2002. Marine biodiversity hotspots and conservation priorities for tropical reefs. Science 295:1280-1284.

Rodgers, J. L. 1999. The bootstrap, the jacknife, and the randomization test: a sampling taxonomy. Multivariate Behavioral Research 34:441-456.

Soberón, J., R. Jiménez, J. Golubov y P. Koleff. 2007. Assessing completeness of biodiversity databases at different spatial 
scales. Ecography 30:152-160.

Sokal, R. R. y F. J. Rohlf. 1995. Biometry, 3rd edition. Freeman, San Francisco. 541 p.

Spalding, M. D., H.E. Fox, G. R. Allen, N. Davidson, Z. A. Ferdaña, M. Finlayson, B. S. Halpern, M. A. Jorge, A. Lombana, S. A. Lourie, K. D. Martin, E. McManus, J. Molnar, C. A. Recchia y J. Robertson. 2007. Marine ecoregions of the world: a bioregionalization of coastal and shelf areas. Bioscience 57:573-583.

Vermeij, G. J. 1999. The accumulation of taxonomic knowledge: the history of species descriptions of some predatory gastropods. Malacologia 41:147-150.

Willott, S. J. 2001. Species accumulation curves and the measure of the sampling effort. Journal of Applied Ecology 38:484486.

Wilson, E. O. 1988. Current state of biological diversity. In Biodiversity, E. O. Wilson (ed.), Smithsonian Institution/ National Academy of Sciences, Washington, DC. p. 3-20.

Whittaker, R.H. 1972. Evolution and measurement of species diversity. Taxon 21:213-251. 


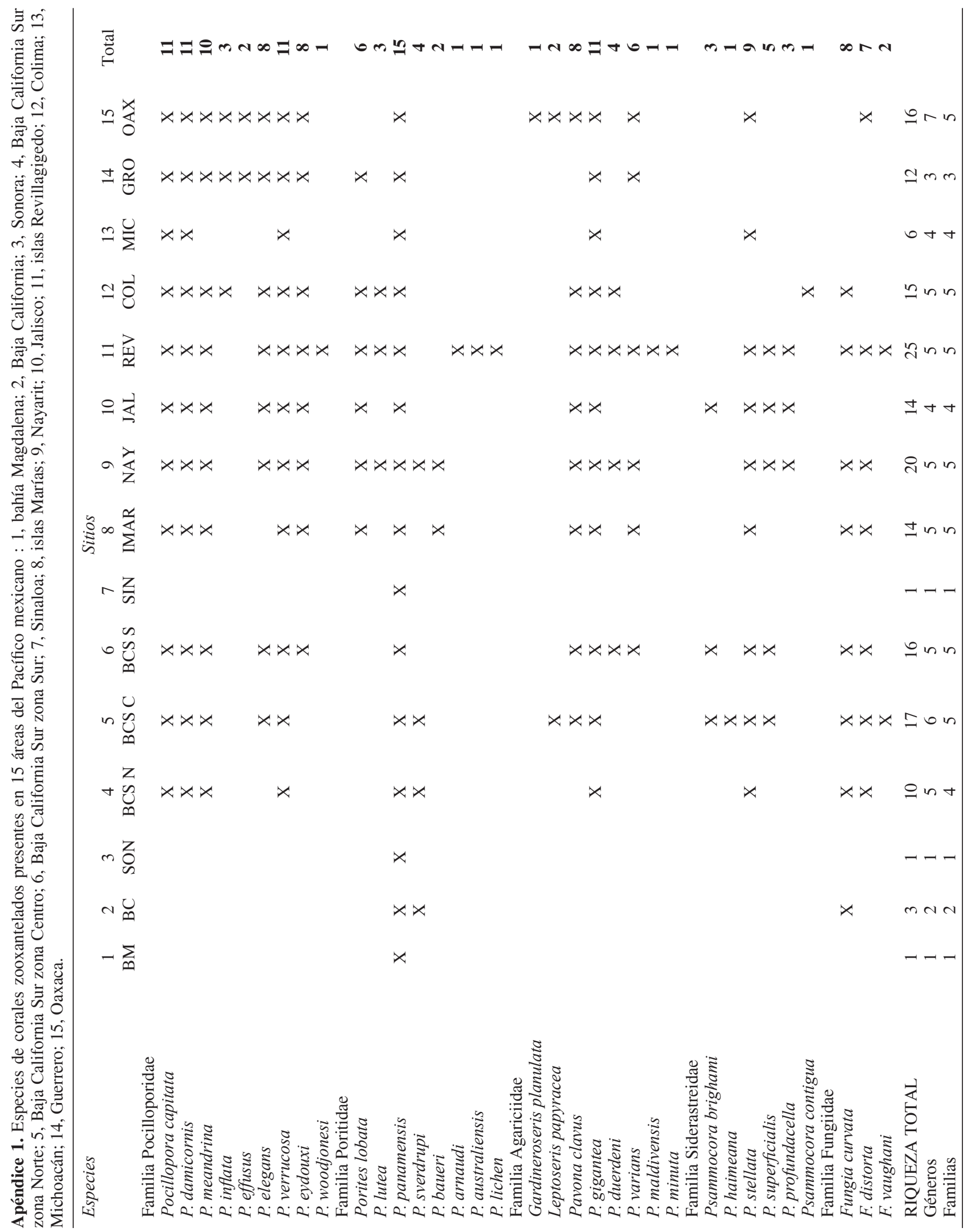

